\title{
Efficacy assessment of pemetrexed treatment of an NSCLC case with brain metastasis
}

\author{
JING LIANG, XIAOLIN LIU, BEIBEI YIN, HAIRONG LIU, JUNJUAN XIAO and YAN LI \\ Department of Oncology, Qianfoshan Hospital, Shandong University, Jinan, Shandong 250014, P.R. China
}

Received May 10, 2012; Accepted August 7, 2012

DOI: $10.3892 / \mathrm{ol} .2012 .888$

\begin{abstract}
Non-small cell lung cancers (NSCLCs) are among the most common malignancies. Although pemetrexed is often used clinically to cure cancers, its efficacy in NSCLC patients with progressive brain metastases remains unclear. Here, we report a successful NSCLC (adenocarcinoma) case treated with pemetrexed. The detected tumors were treated with $900 \mathrm{mg}$ of pemetrexed disodium $\left(500 \mathrm{mg} / \mathrm{m}^{2}\right)$ was administered to the patient on day 1 , and $40 \mathrm{mg}$ of cisplatin $\left(25 \mathrm{mg} / \mathrm{m}^{2}\right)$ was administered on days 1-3, at the interval of 3 weeks. After two cycles of chemotherapy, the brain metastases were reduced. The lesion in the lung was reduced as determined by chest CT-scan. Our results suggest that pemetrexed is an effective therapy for patients with NSCLC and progressive brain metastases.
\end{abstract}

\section{Introduction}

Lung cancer, one of the most common malignancies, is a major cause of morbidity and mortality throughout the world. Approximately $80 \%$ of diagnosed lung cancers are non-small cell lung cancers (NSCLCs). In approximately $20-40 \%$ of NSCLC cases, metastasis to the brain occurs (1-3).

Pemetrexed, a multi-targeted antifolate drug, has been demonstrated in vitro to inhibit at least three different enzymes involved in the human folate pathway including thymidylate synthase (TS), dihydrofolate reductase (DHFR) and glycinamide ribonucleotide formyl transferase $(4,5)$. These enzymes are involved in the synthesis of nucleotides, and ultimately pemetrexed is capable of interfering with RNA and DNA synthesis procedures (6). It was revealed that pemetrexed had a slightly higher brain penetration capability than methotrexate by the analysis of arterial blood and frontal cortex microdialysis samples obtained simultaneously $(7,8)$.

Correspondence to: Professor Yan Li, Department of Oncology, Qianfoshan Hospital, Shandong University, 66 Jingshi Road, Jinan, Shandong 250014, P.R. China

E-mail: doctorlotus75@yahoo.cn

Key words: pemetrexed, non-small cell lung cancer, efficacy, brain metastasis
However, it remains unclear whether penetrexed is effective in NSCLC patients with progressive brain metastases. In the present study we report a successful case of pemetrexed treatment. Patient consent was obtained from the patient and the patient's family.

\section{Case report}

A 46-year-old male patient presented with persistent pain in the left side of his neck, accompanied by low fever. An enlarged lymph node in the left side of the neck was subsequently discovered, which cervical magnetic resonance imaging (MRI) revealed to be a metastatic tumor. A mass $(2.8 \times 1.2 \mathrm{~cm})$ was further visualized by chest computed tomography (CT), and was found to be localized in the posterior segment of the upper right lung. Multiple enlarged lymph nodes in the mediastinum were also detected. Multiple bone and brain metastases were detected by ECT and PET-CT, respectively. The primary lung cancer was diagnosed by histopathologic analysis, electronic bronchoscopy and lung biopsy which revealed a poorly differentiated adenocarcinoma. Although the patient had limited mobility in his neck, the rest of the examinations were normal. Potential mutations on the epithelial growth factor receptor (EGFR) and K-ras genes, obtained from the left cervical lymph node biopsy, were analyzed by DNA sequencing. It was revealed that exon 21 of the EGFR gene contained a heterozygous $T$ to $G$ mutation at nucleotide 2573. However, no mutations were found in the K-ras gene.

To treat the detected tumors, $900 \mathrm{mg}$ of pemetrexed disodium (500 mg/m²; Eli Lilly, Indianapolis, IN, USA) was administered to the patient on day 1 , and $40 \mathrm{mg}$ of cisplatin (25 mg/m²; QILU Pharma., Jinan, China) was administered on days 1-3, with an interval of 3 weeks. Cervical radiotherapy (total dose, 4,200 cGy) was initiated simultaneously. Cetuximab (Merck, Whitehouse Station, NJ, USA) was injected during the second cycle of chemotherapy. Cetuximab $\left(400 \mathrm{mg} / \mathrm{m}^{2}\right)$ was administered on the first day, with weekly injections of $250 \mathrm{mg} / \mathrm{m}^{2}$ cetuximab thereafter. The treatment was terminated after 3 months.

After two cycles of chemotherapy, the brain metastases were reduced as revealed by brain MRI (Fig. 1). The lesion in the lung was reduced as determined by chest CT scans (Fig. 2). The systemic chemotherapy combined with the cetuximab injection was continued. After six cycles of treatment, 
A

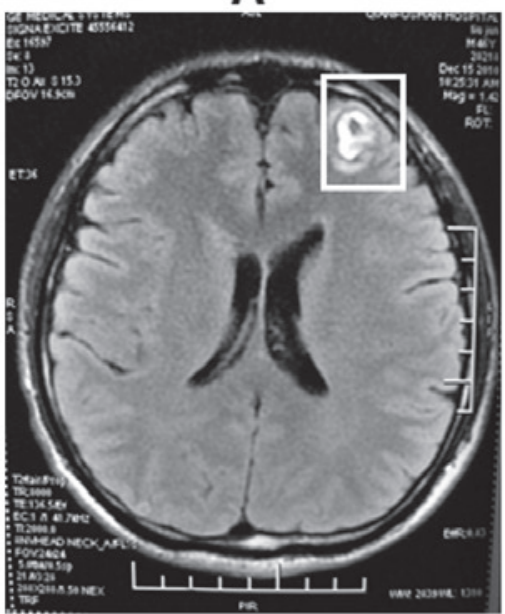

B

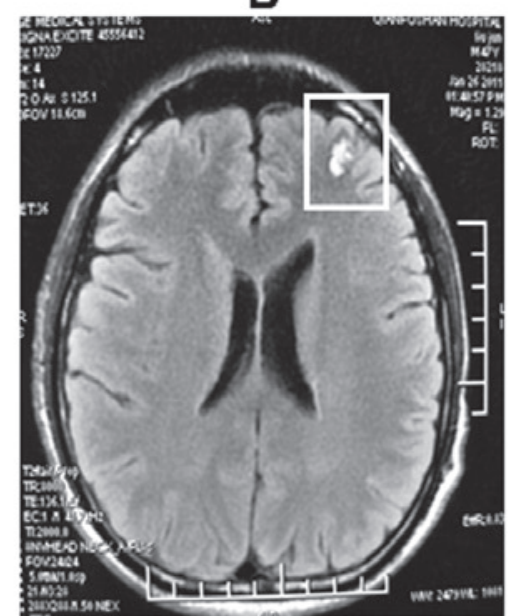

C

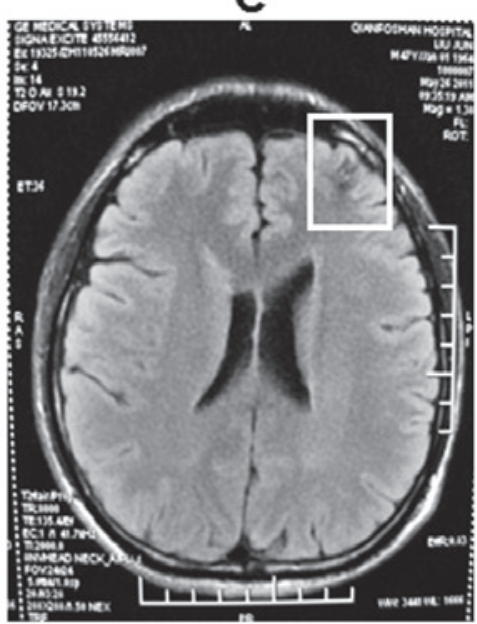

Figure 1. Brain computed tomography (CT) images. (A) Brain CT prior to chemotherapy. (B) Brain CT following two cycles of chemotherapy. (C) Brain CT following six cycles of chemotherapy.

A

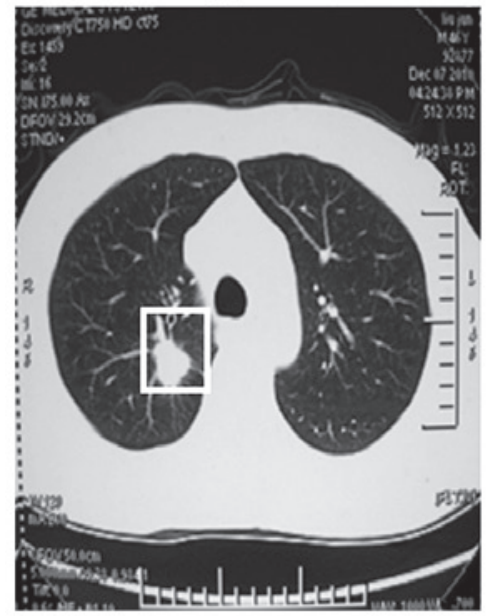

B

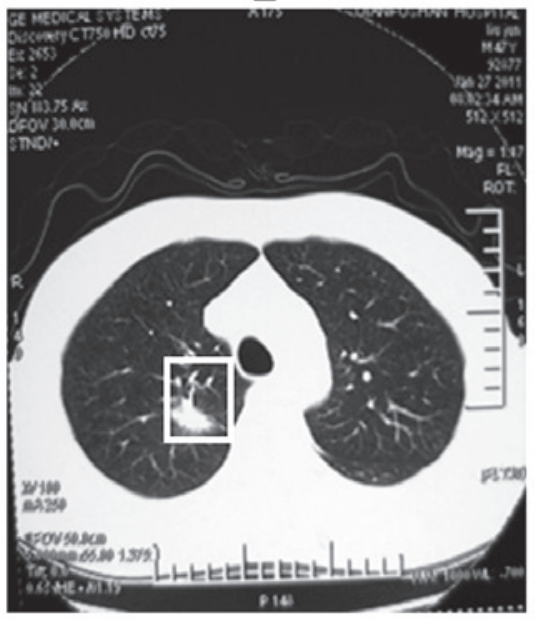

C

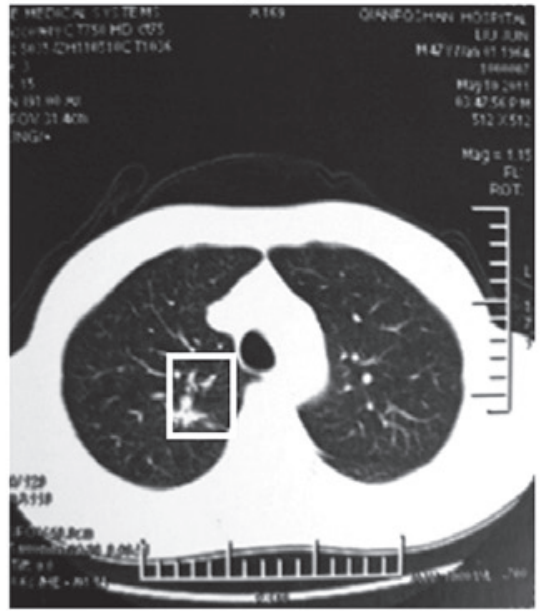

Figure 2. Chest computed tomography (CT) images. (A) Chest CT prior to any chemotherapy. (B) Chest CT following two cycles of chemotherapy. (C) Chest CT following six cycles of chemotherapy.

a PET-CT scan was performed to examine the therapeutic efficacy. There was no abnormal FDG uptake in the brain or the lung. As a result, $900 \mathrm{mg}$ of pemetrexed $\left(500 \mathrm{mg} / \mathrm{m}^{2}\right.$; Eli Lilly) was administered again every 3 weeks. Following the treatments described above, the tumor sizes were significantly decreased (Figs. 1 and 2).

\section{Discussion}

Lung cancer, one of the most common malignant tumors, is a major cause of morbidity and mortality. Approximately $80 \%$ of patients with lung cancers are diagnosed with nonsmall cell lung cancers (NSCLCs). Approximately 20-40\% of NSCLC eventually metastasizes to the brain (1-3).

Pemetrexed, an antifolate drug, is known to inhibit at least three different proteins involved in the human folate pathway including thymidylate synthase (TS), dihydrofolate reductase
(DHFR), and glycinamide ribonucleotide formyl transferase $(4,5)$. These enzymes are involved in the synthesis of nucleotides, and ultimately pemetrexed is capable of interfering with RNA and DNA synthesis (6). Pemetrexed, in combination with cisplatin, has recently been used in the treatment of metastatic NSCLC patients (9-11). NSCLC patients with the exon 19 deletion have a distinctive pattern of brain metastases which usually present as multiple small metastases with low levels of brain edema $(12,13)$. Certain studies have indicated that gefitinib and erlotinib penetrate into the central nervous system and elicit responses in NSCLC patients with brain metastases (13-15). It was revealed that pemetrexed had a slightly higher brain penetration capability compared to that of methotrexate, by the analysis of arterial blood and frontal cortex microdialysis samples obtained simultaneously $(7,8)$. Bearz et al (16) investigated the therapeutic effect of pemetrexed on reducing brain metastases and revealed that PR 
(partial remission) was observed in 11 patients $(28.2 \%)$ and SD (stable disease) was observed in 21 patients $(53.8 \%)$. The total beneficiary rate was $82 \%$ (16). In our case, pemetrexed was proven to be effective even following the failure of radiotherapy. Our study suggests that pemetrexed is an effective therapy for patients with NSCLC (adenocarcinoma) and progressive brain metastases.

\section{References}

1. Adamo V, Franchina T, Adamo B, Scandurra G and Scimone A: Brain metastases in patients with non-small cell lung cancer: focus on the role of chemotherapy. Ann Oncol 17: 1173-1175, 2006.

2. Kawabe T, Phi JH and Yamamoto M: Treatment of brain metastasis from lung cancer. Prog Neurol Surg 25: 148-155, 2012.

3. Mulvenna P, Barton R and Wilson P: Survival of patients with non-small cell lung cancer and brain metastases. Clin Oncol (R Coll Radiol) 23: 375-376, 2011

4. Shih C, Chen VJ, Gossett LS, Gates SB, MacKellar WC and Habeck LL: LY231514, a pirrolo [2,3-d] pyrimidine-based antifolate that inhibits multiple folate-requiring enzymes. Cancer Res 57: 1116-1123, 1997.

5. Barlesi F, Gervais R, Lena H, Hureaux J, Berard H and Paillotin D: Pemetrexed and cisplatin as first-line chemotherapy for advanced non-small-cell lung cancer (NSCLC) with asymptomatic inoperable brain metastases: a multicenter phase II trial (GFPC 07-01). Ann Oncol 22: 2466-2470, 2011.

6. Schultz RM, Chen VJ, Bewley JR, Roberts EF, Shih C and Dempsey JA: Biological activity of the multitargeted antifolate, MTA (LY231514), in human cell lines with different resistance mechanisms to antifolate drugs. Semin Oncol 26: 68-73, 1999.

7. Dai H, Chen Y and Elmquist WF: Distribution of the nove antifolate pemetrexed to the brain. J Pharmacol Exp Ther 315: 222-229, 2005

8. Ortuzar W, Hanna N, Pennella E, Peng G, Langer C, Monberg M and Scagliotti G: Brain metastases as the primary site of relapse in two randomized phase III pemetrexed trials in advanced nonsmall-cell lung cancer. Clin Lung Cancer 13: 24-30, 2012.
9. Schuette W, Tesch H, Büttner H, Krause T, Soldatenkova V and Stoffregen C: Second-line treatment of stage III/IV non-smallcell lung cancer (NSCLC) with pemetrexed in routine clinical practice: Evaluation of performance status and health-related quality of life. BMC Cancer 12: 14, 2012.

10. Azzoli CG, Temin S, Aliff T, Baker S Jr, Brahmer J, Johnson DH and Laskin JL: 2011 Focused update of 2009 American Society of Clinical Oncology clinical practice guideline update on chemotherapy for stage IV non-small-cell lung cancer. J Clin Oncol 29: 3825-3831, 2011.

11. Galetta D, Pisconti S, Cinieri S, Pappagallo GL, Gebbia V and Borsellino N: Induction pemetrexed and cisplatin followed by maintenance pemetrexed versus carboplatin plus paclitaxel plus bevacizumab followed by maintenance bevacizumab: a quality of life-oriented randomized phase III study in patients with advanced non-squamous non-small-cell lung cancer (ERACLE). Clin Lung Cancer 12: 402-406, 2011.

12. Sekine A, Kato T, Hagiwara E, Shinohara T, Komagata T and Iwasawa T: Metastatic brain tumors from non-small cell lung cancer with EGFR mutations: Distinguishing influence of exon 19 deletion on radiographic features. Lung Cancer 77: 64-69, 2012.

13. Jamal-Hanjani M and Spicer J: Epidermal growth factor receptor tyrosine kinase inhibitors in the treatment of epidermal growth factor receptor-mutant non-small cell lung cancer metastatic to the brain. Clin Cancer Res 18: 938-944, 2012.

14. Pesce GA, Klingbiel D, Ribi K, Zouhair A, von Moos R and Schlaeppi M: Outcome, quality of life and cognitive function of patients with brain metastases from non-small cell lung cancer treated with whole brain radiotherapy combined with gefitinib or temozolomide. A randomised phase II trial of the Swiss Group for Clinical Cancer Research (SAKK 70/03). Eur J Cancer 48: 377-384, 2012

15. Heon S, Yeap BY and Britt GJ: Development of central nervous system metastases in patients with advanced non-small cell lung cancer and somatic EGFR mutations treated with gefitinib or erlotinib. Clin Cancer Res 16: 5873-5882, 2010.

16. Bearz A, Garassino I, Tiseo M, et al: Activity of Pemetrexed on brain metastases from non-small cell lung cancer. Lung Cancer 68: $264-268,2010$ 